\title{
High Rates of Fatality Due to AIDS without Universal HIV Testing
}

Shiva Shaan Bassi ${ }^{1}$, J Martin Bland ${ }^{2}$ and Fabiola Martin ${ }^{3^{*}}$

1Department of Biology, University of York, UK

2Department of Health Sciences, University of York, York, UK

3Department of HIV Medicine, Centre for Immunology and Infection, Hull York Medical School, Department of Biology, University of York \& York Teaching Hospital, UK

\begin{abstract}
We audited the cause of death and surrogate markers of HIV patients living in a HIV low prevalence area of the UK. Fatality rates from 2001-2010 were compared to two high prevalence areas. 16/104 newly diagnosed patients died. The median time from diagnosis to death was 4.2 years ( $<1$ month-18 years): $6(38 \%)$ died $<12$ months of diagnosis, $3(19 \%)$ within 5 years, seven $(44 \%)>10$ years later; twelve $(75 \%)$ were male, twelve $(75 \%)$ Caucasian, eight $(50 \%)$ were infected through heterosexual intercourse; median CD4 count was 44 cells/microL (range 2-475). Twelve $(75 \%)$ patients died of AIDS, 4 (25\%) of Pneumocystis jerovecii pneumonia. The observed case-fatality rate was $2.3(95 \%$ $\mathrm{Cl} 1.4-3.9)$ fold higher in this low prevalence area compared to high prevalence areas $(p<0.001)$, where universal HIV testing is promoted. It is possible that an early detection of HIV through universal instead of targeted HIV testing in sexually active adults in all areas of the UK could lead to a reduction of premature death due to AIDS.
\end{abstract}

Keywords: HIV; Mortality; Prevalence; Audit; Pneumocystis jerovecii pneumonia

\section{Introduction}

With free and easy access to testing for Human Immunodeficiency Virus (HIV), Highly Active Anti-Retroviral Therapy (HAART) and specialist care, nobody should advance to or die of Acquired Immune Deficiency Syndrome (AIDS). Yet HIV positive people continue to die of AIDS in the UK. After a sharp decline in AIDS related mortality rates following the introduction of protease inhibitors in mid-1990s, the mortality rates plateaued from 2001 to 2011 [1,2]. During this time a total of $5641 \mathrm{HIV}$ positive people died in the UK, while 69287 patients were officially newly diagnosed [1].

The HPA latest report showed a decline in mortality [3], however patients diagnosed late were still 10 times more likely to die of HIV within the first year of detection compared to earlier diagnosis $(4.0 \%$ vs. $0.4 \%)[1,3]$. In the UK, the proportion of undiagnosed HIV positive people has not reduced over the last 10 years [2] and the proportion of those diagnosed late is highest in North and East of the UK [3]. The definition of 'late diagnosis' has changed but essentially means the detection of HIV after a time point when treatment should have been initiated [2]. It has been subcategorised as 'advanced HIV disease' (CD4 count $<200$ cells/microL and/or AIDS-defining illness) or 'late HIV presentation' (CD4 count $<350$ cells/microL and/or AIDS-defining illness) within the first three months of diagnosis [1,4,5]. Advanced HIV disease is not only associated with a poor outcome but it is labour intensive, costly to manage and drives HIV transmission [4,6-8].

The BHIVA 2008 HIV testing guidelines recommend routine HIV testing in people belonging to certain risk groups, presenting with indicator diseases and living in HIV high prevalence areas $(\geq 2$ cases/1000) [9]. Therefore, in low prevalence areas, for people, who do not fall under a risk group, testing is only recommended when they are symptomatic with HIV, which apart from sero-conversion syndrome is associated with advanced disease. Considering that the bulk of UK's population live in HIV low prevalence areas, a large number of people will never be tested. However HIV prevalence has been increasing steadily, especially in Yorkshire \& The Humber (Y\&TH), an HIV low prevalence area. It is estimated that by 2015 the number of people accessing HIV care in Y\&TH will be 5300 , which will be $61 \%$ greater than in 2009 [10].
We report the outcome of a mortality audit of HIV positive patients in York. It was felt that it was possible that HIV case fatality was higher in HIV low prevalence than high prevalence areas, since asymptomatic HIV carriers not belonging to a HIV risk group, when living in HIV low prevalence area, are exempt from routine HIV testing. We therefore compared the local HIV fatality rate with two high prevalence areas of London over a period of 10 years.

\section{Methods}

This audit was conducted as part of the clinical governance at York Teaching Hospital from January 2001-December 2010. The hospital's audit department and the lead clinician for Research \& Development did not require ethical approval for this audit to be conducted and published. Outcome measures were: number and cause of deaths, number of late diagnosis, time of HIV-1 diagnosis to death, place of death, CD4 count and HIV-1 proviral load at time of diagnosis/death and antiretroviral treatment.

Published and specifically requested Health Protection Agency data on HIV mortality, HIV new diagnosis and death concentrating on South-East London, North-West London (SE, NW London), Y\&TH and York were reviewed (2001-2011). Detailed causes of death are withheld to preserve patient anonymity.

\section{Statistical analysis}

The expected number of deaths for the York area were calculated, assuming that the ratio of deaths to new diagnoses were the same as for London areas (2001-2010). This was compared to the observed deaths

*Corresponding author: Fabiola Martin, Senior Clinical Lecturer in HIV Medicine, Centre for Immunology and Infection, University of York, Wentworth Way, York, YO10 5DD, UK, Tel: 01904 328907; Fax: 01904 328844; E-mail: Fabiola.Martin@hyms.ac.uk

Received December 20, 2013; Accepted February 26, 2014; Published March 10,2014

Citation: Bassi SS, Bland JM, Martin F (2014) High Rates of Fatality Due to AIDS without Universal HIV Testing. J AIDS Clin Res 5: 291. doi:10.4172/2155 6113.1000291

Copyright: (C) 2014 Bassi SS, et al. This is an open-access article distributed under the terms of the Creative Commons Attribution License, which permits unrestricted use, distribution, and reproduction in any medium, provided the original author and source are credited. 
using a chi-squared test. A confidence interval for the ratio of observed to expected deaths was calculated using the exact Poisson distribution.

\section{Results}

\section{HIV deaths in York, 2001-2010}

HIV was diagnosed in 104 patients and 16 HIV patients died. The demographics are listed in Table 1 . The median time from diagnosis to death was 4.2 years (range $<1$ month to 18 years); six (38\%) patients died within 12 months of diagnosis, three (19\%) within 5 years and seven $(44 \%)>10$ years later.

Twelve/16 (75\%) patients were male, 12/16 (75\%) of Caucasian ethnicity and the mean age at diagnosis was 40 (median 38 , range 23-65). Eight/16 (50\%) patients were infected through unprotected heterosexual intercourse. Median CD4 count at time of diagnosis were available for 11 patients: 38 cells/microL (2-400); and at time of death for all 16 patients: 44 cells/microL (2-475). Nine patients were late presenters with a CD4 count $<350$ cells/microL of whom $6 / 9$ patients had a CD4<200 cells/microL (median 30 cells/microL (254)). All patients with advanced HIV disease died within 12 months of diagnosis. Median HIV-1 viral load was available for seven patients at time of diagnosis: 190000 (23300-340000) copies/L; and for 13 patients at time of death: $100000\left(0-1.9 \times 10^{6}\right)$ copies/L.

Of the 16 patients, three (18.8\%) initiated HAART within three months of diagnosis, one (6.2\%) within a year and eight (50\%) more than a year after diagnosis. These eight individuals were all diagnosed before the HAART era. Four (25\%) patients had not initiated antiretroviral treatment, of whom three died within one month of diagnosis. The one other patient refused treatment. Thirteen $(80 \%)$ patients died in the hospital, two in the community and data were unavailable for one patient.

Cause of death was known for 16 patients: Twelve (75\%) patients died directly as a consequence ofAIDS through opportunistic bacterial or fungal co-infections, non-Hodgkin lymphomas and HIV associated dementia. The most common opportunistic infection was Pneumocystis jerovecii pneumonia (PJP), 4/16 (25\%). Seven (44\%) patients died

\begin{tabular}{|l|c|c|c|}
\hline Men & Number & Median & Range \\
\hline Ethnicity & $12(75 \%)$ & & \\
\hline \multicolumn{1}{|c|}{ Caucasian } & $12(75 \%)$ & & \\
\hline Black African & $4(25 \%)$ & & \\
\hline Risk-factor & & & \\
\hline \multicolumn{1}{|c|}{ Heterosexual } & $8(50 \%)$ & & \\
\hline \multicolumn{1}{|c|}{ MSM } & $3(19 \%)$ & & \\
\hline \multicolumn{1}{|c|}{ Other } & $5(31 \%)$ & & \\
\hline Age at diagnosis (years) & & 38 & $23-65$ \\
\hline Age at death (years) & & 46.50 & $31-80$ \\
\hline Time from diagnosis to death (years) & & 4.20 & $0.04-17.82$ \\
\hline CD4 at diagnosis (cells/microL) & & 38 & $9-400$ \\
\hline CD4 at death (cells/microL) & & 44 & $2-475$ \\
\hline VL at diagnosis (copies/L) & & 19000 & $23300-340000$ \\
\hline VL at death (copies/L) & & 100000 & $0-1.9 \times 10^{6}$ \\
\hline
\end{tabular}

Table 1: Demographics and surrogate markers of HIV positive patients diseased in York.

$M S M=$ men having sex with men and VL= HIV viral load

\begin{tabular}{|l|c|c|c|}
\hline & $\begin{array}{c}\text { South East } \\
\text { London }\end{array}$ & $\begin{array}{c}\text { North West } \\
\text { London }\end{array}$ & City of York \\
\hline $\begin{array}{l}\text { Diagnosed HIV prevalence per 1 000, } \\
\text { average (2010, aged 15-59), (\%) }\end{array}$ & $7.13(0.7 \%)$ & $4.95(0.5 \%)$ & $0.51(0.05 \%)$ \\
\hline New HIV diagnosis (2001-2010, all ages) & 6988 & 8971 & 95 \\
\hline First AIDS (2001-2010, all ages) & 927 & 685 & 28 \\
\hline AIDS: New Diagnosis & $1: 8$ & $1: 13$ & $1: 4$ \\
\hline Total Deaths (2001-2010, all ages) & 513 & 550 & 16 \\
\hline
\end{tabular}

Table 2: HIV prevalence, New HIV diagnosis, First AIDS, Deaths among HIV positive people in South East and North West London and City of York.

within 15 months and another two within 5 years of diagnosis. Six patients who died within $<1$ year of AIDS/opportunistic infections had a median CD4 count of 26 cells/microL (2-63) and had been diagnosed after 2006.

Seven (44\%) patients had been diagnosed in the pre-HAART era and all died $>10$ years post diagnosis: three died of AIDS and four died of serious non-AIDS events. The median CD4 count and HIV viral load at time of death were $101(16-286)$ and $43,300\left(0\right.$ to $\left.1.9 \times 10^{6}\right)$. Four patients carried multi-class antiretroviral resistant HIV-1 virus.

Data on recent hospital attendance were available on $4 / 6$ patients who had died within 12 months of diagnosis. All four patients were diagnosed during their acute hospital admission, each individual had attended at least two different hospital departments and two patients also had visited their general practitioners within the previous year of their diagnosis without being offered a HIV test.

\section{Observed vs. expected fatality rate in York}

SE London has an estimated HIV prevalence of 7.13/1000 (95\% CI: 2.05 to 13.88); NW London has a HIV prevalence of $4.95 / 1000$ (95\% CI 1.95 to 8.61 ) and York; Y\&TH has a population of a HIV prevalence of $0.74 / 1000$ (95\% CI 0.25 to 2.00 ) [11,12]. We posed the question: how does the observed number of deaths in York compare to what we would expect if case fatalities were like London area? The ratio of London deaths to new cases is 0.0666 . Given 104 new diagnoses, we would therefore expect $104 \times 0.0666=6.93$ deaths. However the observed 16 deaths are significantly higher $(\mathrm{p}=0.001)$. The magnitude of this difference is expressed by calculating the ratio of observed to expected deaths: $16 / 6.93=2.3$. Hence the estimated risk of death for a person detected with HIV in our low prevalence area is 2.3 (95\% CI 1.33.7) times the risk for a patient detected in London area. An alternative analysis using the logs of the ratios gave similar results: 2.3 (95\% CI 1.4-3.9). Table 2 summarises data on HIV new diagnosis, first AIDS and deaths in London areas and York.

\section{Discussion}

We audited all mortalities of known HIV positive patients at our hospital during an arbitrary set period of time: 2001-2010. A total of 16 people died in 10 years, of whom nine had been diagnosed late and six had advanced disease and died within the first 12 months post diagnosis. Seventy-five per cent of all patients who died were white Caucasian and $50 \%$ heterosexual. Twelve $(75 \%)$ deaths were due to severe immunosuppression caused by HIV, of which four (25\%) were due to PJP.

Nationally, data on the incidence of all cause death within the first year of all HIV diagnosis are not well captured. Data on new HIV diagnosis and total HIV deaths were used to estimate how many people with HIV might have died of late diagnosis over a certain period of time. Compared to the London area, the observed death toll in our HIV 
low prevalence area was 2.3 fold as high as expected. This could be due to low exposure of generalists to AIDS cases, but our audit did not find a systematic failure in care provision, especially since once diagnosed all patients were cared for by HIV inpatient specialists.

Late diagnosis of HIV or AIDS is the most important risk factor for HIV associated mortality $[13,14]$. Death caused by HIV or AIDS is considerably underreported, since $22 \%$ of the HIV positive population remain undiagnosed and so patients may die of AIDS without ever being tested [3]. Although not all deaths in individuals newly diagnosed with HIV are due to AIDS, it is known that two third of deaths occur in patients with HIV late diagnosis $[1,3]$. The most common cause of death remains to be PJP, a preventable disease if HAART with/without PJP prophylaxis is initiated early enough $[1,3]$. Despite good adherence to PJP treatment guidelines, mortality rates remain high, between 35 $40 \%[15,16]$.

BHIVA, MedFaSH HIV and NICE HIV testing and treatment guidelines emphasise the need to diagnose HIV as soon as possible $[9,17,18]$. In low prevalence areas, however, HIV testing is recommended only in certain risk groups and patients with HIV indicator disease. In these areas HIV associated deaths are probably even higher than reported, since patients often are not offered a HIV test, when not falling into risk groups, even if they have indicator diseases. The combination of patients' white ethnicity and heterosexuality together with HIV being classified as rare in certain areas, might prevent patients and clinicians to consider HIV as the underlying and treatable problem [19].

Our observation is reflected in the HPA HIV report 2013 report, where higher rates of one year HIV mortality by CD 4 counts $<350$ were observed: in areas outside of London, in heterosexual men, in people of white ethnicity and older $>50$ years of age [3]. Potentially, medical training to recognize HIV indicator disease is not prioritized over other competing subjects in low prevalence areas. Nevertheless offering a HIV test only to those with an indicator disease, which is mostly associated with advanced disease, means that people are rarely diagnosed when still immune-competent.

Meanwhile the 'opt out HIV testing on admission' has been implemented in several European Union countries for many decades $[20,21]$. In USA and France routine one-time testing has been shown to be cost effective and reduce transmission independent of HIV prevalence [22]. The US Preventive Services Task Force (USPSTF) guidelines recommend routine HIV screening for every US resident aged 15-65 [23]. Das and Volberding's editorial summarizes clearly the move from risk groups targeted to population based HIV testing, which ultimately increases the life expectancy of HIV positive patients and decreases HIV transmission through timely HAART initiation [24]. Lucas et al. go as far as suggesting three monthly HIV testing in risk groups and shows its cost effectiveness [25].

This audit has several limitations: data sets were partially incomplete, specific data were collected for death in HIV positive patients occurring in York and not for whole of Y\&TH. The strength of this audit is that the data reported for York is accurate.

High fatality rate due to late diagnosis of HIV and AIDS is not limited to high prevalence areas, but is a common feature of all late HIV diagnosis. However it is possible that the mortality outcome of patients with HIV living in a low prevalence area is significantly worse due to lack of universal HIV testing. Rate of transmission per person in a low prevalent area also remains a worry, since these HIV carriers are not considered at risk and can pass on HIV without knowing for at least a decade. We believe that late HIV testing in a population seemingly not at risk of HIV acquisition, is an important contributing factor to premature death and unintended untoward transmission. We hope to test this hypothesis by comparing our data with other HIV mortality audits from low prevalence areas.

\section{Acknowledgements}

We thank Drs lan Fairley, Clare Knowles, Mrs Fiona Wallis, Mr Peter Tovey and Prof Charles Lacey and all medical professionals on the ward and intensive care unit involved in patient care. We thank Dr. Simon Padfield for critical review of the paper and the HPA for data provision.

\section{References}

1. HPA (2011) HIV in the United Kingdom. 2011 report.

2. Kall MM, Smith RD, Delpech VC (2012) Late HIV diagnosis in Europe: a call for increased testing and awareness among general practitioners. Eur J Gen Pract 18: 181-186.

3. HPA (2013) HIV in the United Kingdom. 2013 report.

4. Krentz HB, Auld MC, Gill MJ (2004) The high cost of medical care for patients who present late (CD4 <200 cells/microL) with HIV infection. HIV Med 5: 93-98.

5. Antinori A, Coenen T, Costagiola D, Dedes N, Ellefson M, et al. (2011) Late presentation of HIV infection: a consensus definition. HIV Med 12: 61-64.

6. Attia S, Egger M, Müller M, Zwahlen M, Low N (2009) Sexual transmission of HIV according to viral load and antiretroviral therapy: systematic review and meta-analysis. AIDS 23: 1397-1404.

7. May M, Gompels M, Delpech V, Porter K, Post F, et al. (2011) Impact of late diagnosis and treatment on life expectancy in people with HIV-1: UK Collaborative HIV Cohort (UK CHIC) Study. BMJ 343: d6016.

8. Marks G, Crepaz N, Senterfitt JW, Janssen RS (2005) Meta-analysis of highrisk sexual behavior in persons aware and unaware they are infected with HIV in the United States: implications for HIV prevention programs. J Acquir Immune Defic Syndr 39: 446-453.

9. BHIVA (2008) UK National Guidelines for HIV Testing 2008.

10. Public Health England (2011) NHS Yorkshire and the Humber QIPP resource pack: January 2011 (Sexual Health)

11. HPA (2010) Diagnosed HIV prevalence in Local Authorities (LAs) in England, 2010.

12. HPA (2010) HIV in the United Kingdom: 2010 report.

13. Chadborn TR, Delpech VC, Sabin CA, Sinka K, Evans BG (2006) The late diagnosis and consequent short-term mortality of HIV-infected heterosexuals (England and Wales, 2000-2004). AIDS 20: 2371-2379.

14. Sabin CA, Smith CJ, Gumley H, Murphy G, Lampe FC, et al. (2004) Late presenters in the era of highly active antiretroviral therapy: uptake of and responses to antiretroviral therapy. AIDS 18: 2145-2151.

15. Wang HW, Lin CC, Kuo CF, Liu CP, Lee CM (2011) Mortality predictors of Pneumocystis jirovecii pneumonia in human immunodeficiency virus-infected patients at presentation: Experience in a tertiary care hospital of northern Taiwan. J Microbiol Immunol Infect 44: 274-281.

16. CDC (2013) Pneumocystis pneumonia Statistics

17. NICE (2011) Increasing the uptake of HIV testing among men who have sex with men.

18. MEDFASH (2003) Recommended standards for NHS HIV services.

19. Ellis S, Graham L, Price DA, Ong EL (2011) Offering HIV testing in an acute medical admissions unit in Newcastle upon Tyne. Clin Med 11: 541-543.

20. Hamill M, Burgoine K, Farrell F, Hemelaar J, Patel G, et al. (2007) Time to move towards opt-out testing for HIV in the UK. BMJ 334: 1352-1354.

21. Mounier-Jack S, Nielsen S, Coker RJ (2008) HIV testing strategies across European countries. HIV Med 9: 13-19.

22. ECDC (2010) HIV testing Increasing uptake and effectiveness in the European Union: European Centre for Disease Prevention and Control.

23. U.S. Preventive Services Task Force (2013) Screening for HIV. 
Citation: Bassi SS, Bland JM, Martin F (2014) High Rates of Fatality Due to AIDS without Universal HIV Testing. J AIDS Clin Res 5: 291. doi:10.4172/21556113.1000291

Page 4 of 4

24. Das M, Volberding P (2013) Bringing the end in sight: consensus regarding HIV screening strategies. Ann Intern Med 159: 63-64.
25. Lucas A, Armbruster B (2013) The cost-effectiveness of expanded HIV screening in the United States. AIDS 27: 795-801. 